\title{
Fault Diagnosis Technology of Intelligent Substation UPFC System Based on Abnormal Sub-module Port Voltage
}

\author{
Jinghong Zhao ${ }^{1}$, Lei Jin ${ }^{1}$, Fanbo Meng ${ }^{1}$, Xin Hong ${ }^{1}$, Xing Huang ${ }^{{ }^{*}}$ \\ ${ }^{1}$ State Grid Liaoning Electric Power Supply Co., LTD, Shenyang 110006 China
}

\begin{abstract}
Unified power flow controller (UPFC) combines the advantages of FACTS devices of series and parallel, which is an important direction for the development of intelligent substations in the future. However, a large number of MMC submodules have become potential fault points of UPFC system. The rapid judgment of fault state and accurate positioning of fault sub-modules are the primary conditions to ensure the safe and stable operation of UPFC system, which can effectively reduce the mobile operation and maintenance time of power engineering operation and maintenance personnel, and guarantee the safety of operation and maintenance. Therefore, this paper proposes a fault diagnosis technology of intelligent substation UPFC system based on abnormal sub-module port voltage (SPVE). Experimental results show that the SPVE method has advantages in identification accuracy and rapidity.
\end{abstract}

\section{Introduction}

In China, the distribution of energy and economic development is extremely unbalanced. Large energy bases are mostly concentrated in northwest and northeast China, with low local load level and large surplus of electric power. The load centers are mainly concentrated in the economically developed southeast coastal areas, and the power supply is limited. In order to ensure the full utilization of electric energy in energy bases and alleviate the power supply shortage in load centers, the electric energy in large energy bases must be transmitted across multiple regional grids for a long distance. However, considering the difference of the transmission and receiving double-end power network, a series of problems such as low-frequency shock and power shock will occur if the connection of the power network is carried out directly.

Unified power flow controller (UPFC) combines the advantages of FACTS devices of series and parallel, which is an important direction for the development of intelligent substations in the future. However, a large number of MMC submodules have become potential fault points of UPFC system. The rapid judgment of fault state and accurate positioning of fault sub-modules are the primary conditions to ensure the safe and stable operation of UPFC system, which can effectively reduce the mobile operation and maintenance time of power engineering operation and maintenance personnel, and guarantee the safety of operation and maintenance. Therefore, this paper proposes a fault diagnosis technology of intelligent substation UPFC system based on abnormal sub-module port voltage (SPVE).
Experimental results show that the SPVE method has advantages in identification accuracy and rapidity.

\section{UPFC system modeling}

Figure 1 shows the structure of UPFC system. Considering the voltage rating limitation of power electronic equipment in the UPFC device, transformers are usually installed at both ends of the converter 1 and 2, as shown in the figure 1(a). Both converter 1 and 2 adopt MMC topology, as shown in the figure 1(b). According to Kirchhoff's current law, when MMC topology operates under normal working conditions, there exists the following relationship among bridge arm current, ac side current and circulation current:

$$
i_{x, \mathrm{u} / 1}= \pm \frac{i_{\mathrm{s}, x}}{2}+i_{z, x}
$$

Where $u$ and 1 represent upper and lower bridge arms of each phase; $i_{\mathrm{s}, \mathrm{x}}$ is the ac side current; $i_{\mathrm{s}, \mathrm{x}}$ is the internal circulation of MMC topology.

Due to the complex topological structure of MMC and the large number of sub-modules, in order to facilitate analysis, the system model needs to be simplified, and each bridge arm is equivalent to a variable voltage source $\left(v_{\mathrm{x}, \mathrm{u}}, v_{\mathrm{x}, 1}\right)$, as shown in figure 2 . The output voltage of each bridge arm is determined by the switching state of its subsidiary modules.

$$
v_{x, \mathrm{u} / 1}=\sum_{m=1}^{\mathrm{n}} S_{x, m, \mathrm{u} / 1} \frac{\mathrm{V}_{\mathrm{dc}}}{\mathrm{n}}
$$

Where $S_{x, m, \mathrm{u} / 1}=0 / 1$ is the switching state of submodule; $m=1,2, \ldots n$ is the serial number of each bridge arm sub-module. 


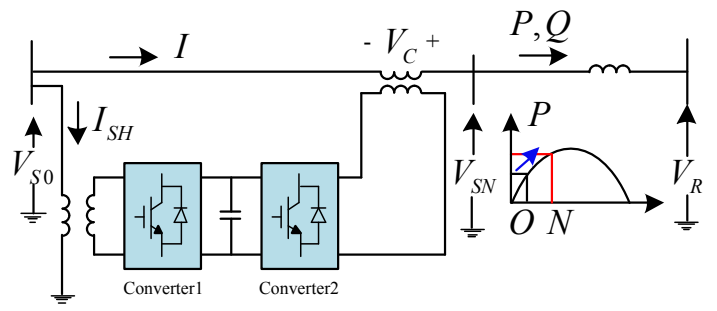

(a)

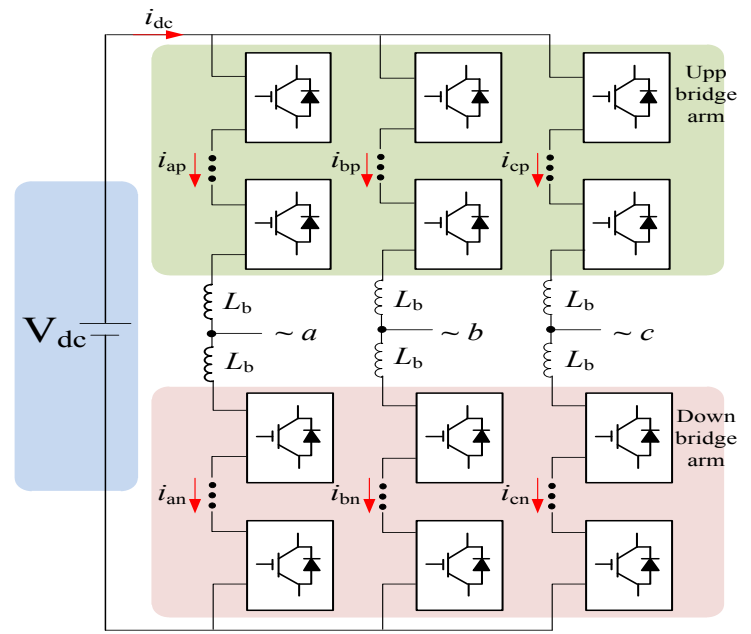

(b)

Figure 1. The simplified circuit of UPFC system.

According to the modeling results, the switching state of submodules not only directly determines the ac side voltage and current of $\mathrm{MMC}$, but also has a strong coupling relationship with each phase circulation. Therefore, by planning the switching state of submodules of each bridge arm, collaborative control of the output voltage, current, capacitor voltage of submodules and internal circulation of the $\mathrm{MMC}$ converter can be achieved.
UPFC topology adopts series structure of multiple submodules, which can effectively solve the problem of voltage resistance of power devices and provide better output quality for the system.

However, since the semiconductor power switch device is one of the most frequently malfunctioning components of the converter system, each sub-module is the potential failure point of the UPFC system. As shown in figure 2, sub-module faults are generally divided into short-circuit faults and open-circuit faults.

\section{UPFC fault status analysis}

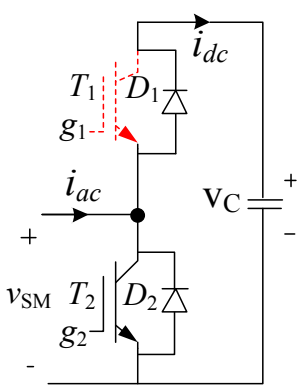

(a) $T_{1}$ open circuit fault

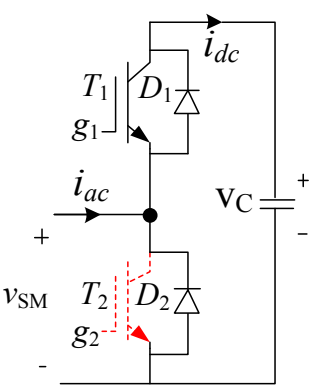

(b) $T_{2}$ open circuit fault

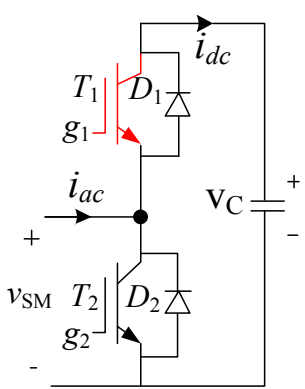

(c) $T_{1}$ short circuit fault (d) $T_{2}$ short circuit fault

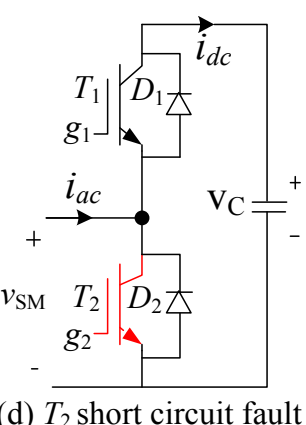

Figure 2. The typical fault of UPFC system.

In figure 2 , if an open circuit fault occurs in a submodule of the UPFC system, the output voltage of the fault submodule can be expressed as

$T_{1}$ open circuit fault:

$$
\begin{aligned}
& S_{\mathrm{sm}}=1: \quad v_{\mathrm{sm}}=\left\{\begin{array}{l}
\mathrm{v}_{\mathrm{c}} ; i_{\mathrm{arm}}>0 \\
0 ; i_{\mathrm{arm}}<0
\end{array}\right. \\
& S_{\mathrm{sm}}=0: \quad v_{\mathrm{sm}}= \begin{cases}0 ; i_{\mathrm{arm}}>0 \\
0 ; i_{\mathrm{arm}}<0\end{cases}
\end{aligned}
$$

$T_{2}$ open circuit fault: 


$$
\begin{aligned}
& S_{\mathrm{sm}}=1: \quad v_{\mathrm{sm}}=\left\{\begin{array}{l}
\mathrm{v}_{\mathrm{c}} ; i_{\mathrm{arm}}>0 \\
\mathrm{v}_{\mathrm{c}} ; i_{\mathrm{arm}}<0
\end{array}\right. \\
& S_{\mathrm{sm}}=0: \quad v_{\mathrm{sm}}=\left\{\begin{array}{l}
\mathrm{v}_{\mathrm{c}} ; i_{\mathrm{arm}}>0 \\
0 ; i_{\mathrm{arm}}<0
\end{array}\right.
\end{aligned}
$$

Similarly, short circuit fault phenomena of $T_{1}$ and $T_{2}$ can be analyzed, which will not be repeated here due to the limitations of the article.

\section{Fault detection and diagnosis technology based on SPVC}

The fault diagnosis method of UPFC system based on SPVE is divided into two parts: fault detection and diagnosis (FDD). The FDD system designs a state observer to observe the state variables of the UPFC system, so as to judge whether the system is running in a healthy state.

\subsection{Condition monitoring}

Considering the strong coupling and quasi-linear relationship among system variables such as ac side current, bridge arm port voltage and submodule capacitance voltage of UPFC system, extended kalman filter (EKF) is adopted to monitor the fault state of UPFC system. Based on the discrete domain mathematical model of UPFC system, EKF observer obtains the observed state variables by using the state estimation value, the input variable and the measured state variable at the current moment. The general form of the extended kalman filter nonlinear equation of state can be expressed as

$$
\left\{\begin{array}{l}
\mathrm{d} \hat{x}=\mathbf{A}(\hat{x}) \cdot \hat{x}+\mathbf{B} \cdot u+\mathbf{G} \cdot(y-\hat{y}) \\
\hat{y}=\mathbf{C} \hat{x}
\end{array}\right.
$$

Where $\hat{x}$ is the state variable of the observed system; $u$ is the input variable; $y$ is the measured quantity and $\hat{y}$ is the observed quantity; $\mathbf{A}$ is the state variable matrix; $\mathbf{B}$ is the input variable matrix; $\mathbf{C}$ is the observation matrix; $\mathbf{G}$ is the gain matrix.

\subsection{Fault location}

When the power switching device of the sub-module has an open circuit or short circuit fault, the control signal generated by the system cannot effectively act on the sub-module of the fault, resulting in a large residual difference between the actual port voltage of the submodule of the fault and the expected port voltage of the sub-module of the system. The residual error and fault state duration of the port voltage sampling value and expected value of the sub-module are taken as evaluation criteria to design the fault location link of the submodule, as shown in figure 3 .

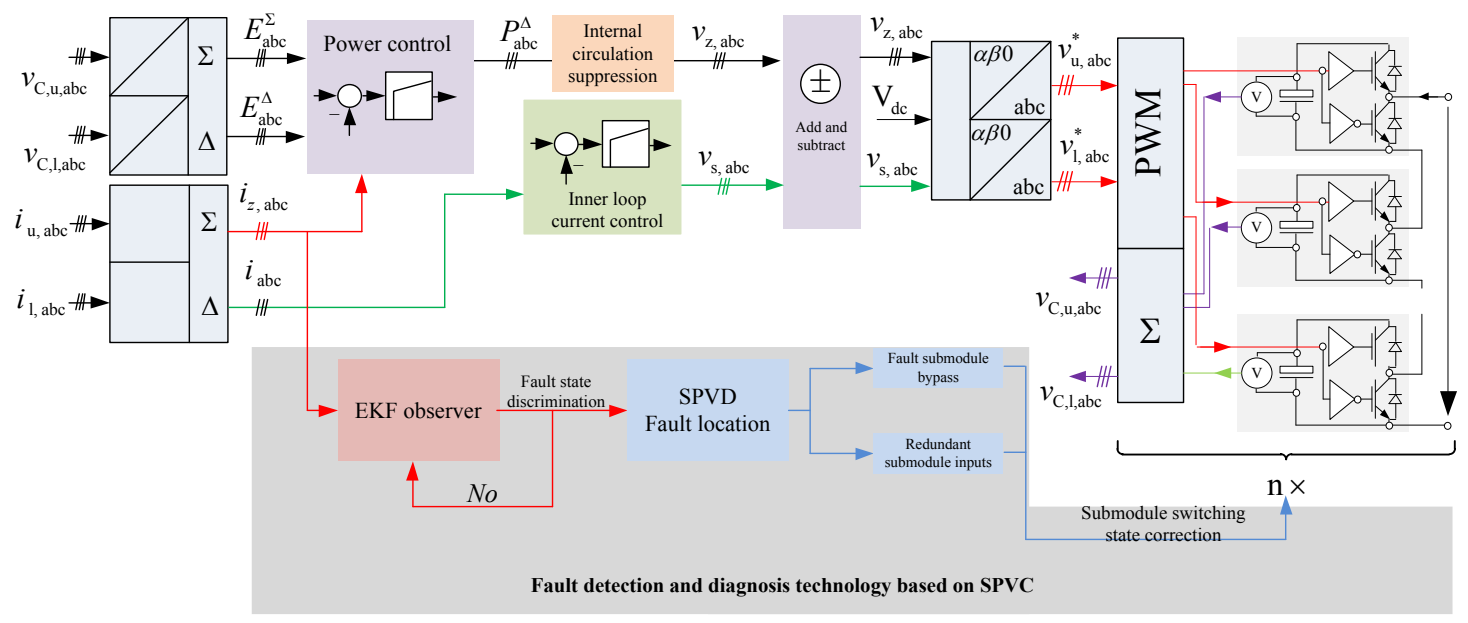

Figure 3. The structure of UPFC fault diagnosis system.

The detailed implementation steps are as follows:

1) By comparing the port voltage of the sample module with the minimum capacitance voltage in healthy working state, the switching state corresponding to the normal working state of the sub-module is obtained. If the port voltage of the sub-module is greater than the minimum capacitance voltage of the sub-module in the healthy working state, the expected switching state is input; otherwise, the expected switching state is bypass.

2) The ideal switching state obtained by inversion and the actual switching state are calculated logically to obtain the current fault judgment signal.
3) In order to prevent misdiagnosis and interference of dead zone, narrow pulse and other phenomena on fault location, the above logical operation results need to be stored. If the number of continuous fault state maintenance cycles exceeds the preset threshold, the fault submodule will be judged and the fault submodule sequence number will be obtained.

\section{Experimental verification and analysis}

In order to verify the correctness and feasibility of the above UPFC system fault diagnosis method, 
experimental verification and analysis were conducted based on the $55 \mathrm{~kW}$ three-phase UPFC system test prototype.

Figure 4 shows the dynamic experimental results of submodule open circuit fault in UPFC system. Before the failure, the residual of EKF circulation observation and measurement converges to 0 . After the submodule open circuit fault occurs, the residual error of the observation circulation is greater than the threshold. After the fault occurs $10 \mathrm{~ms}$, the state monitoring link determines that the system is in the fault state and can preliminarily locate the fault phase unit. The fault diagnosis system quickly enters the positioning link after obtaining the fault state signal. Topological sub module as shown in the figure below, if the UPFC system open-circuit fault occurs, the port voltage and expected output voltage between the residual and failure cycle number is greater than the preset threshold, the fault location link through simple logical operations and state storage can capture fault module serial number, after obtaining the fault signal within $3 \mathrm{~ms}$ after finish the accurate position of fault point.

Similarly, figure 5 shows the dynamic experimental results of sub-module short-circuit fault in UPFC system. When the difference between observed and measured values of UPFC internal circulation also does not converge to zero, the short-circuit fault status monitoring of UPFC system can be realized.

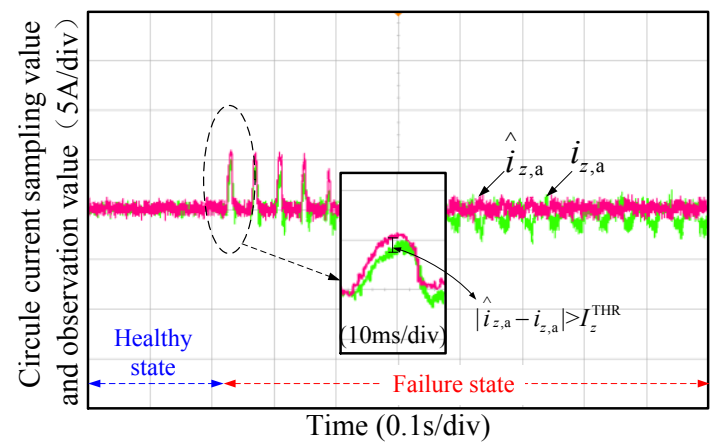

(a)

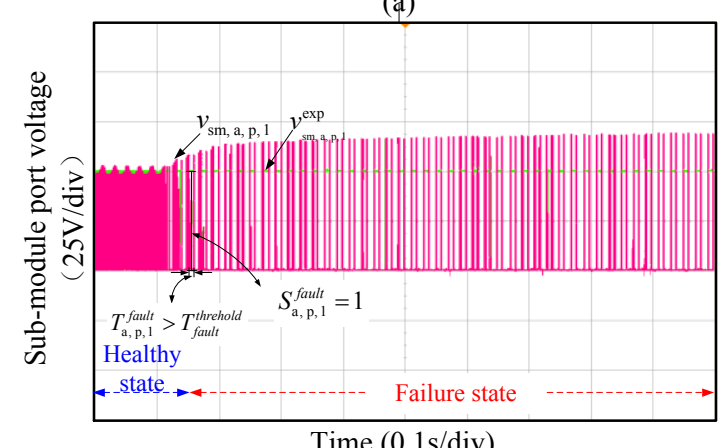

(b)

Figure 4. The results of sub-module open circuit fault diagnosis.

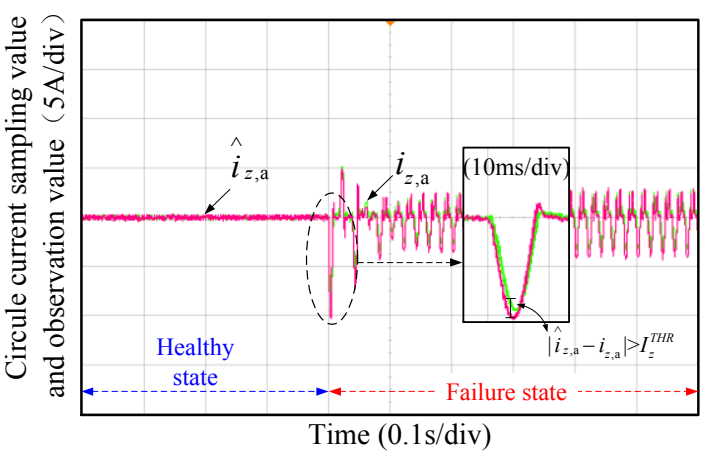

(a)

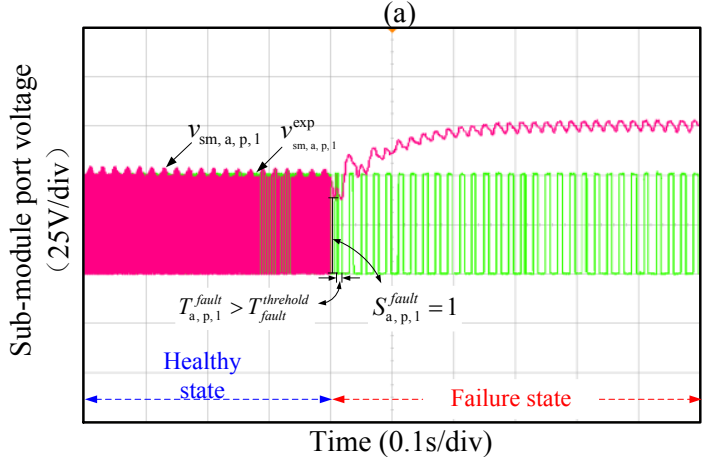

(b)

Figure 5. The results of sub-module short circuit fault diagnosis.

\section{Conclusion}

Unified power flow controller (UPFC) combines the advantages of FACTS devices of series and parallel, which is an important direction for the development of intelligent substations in the future. However, a large number of MMC submodules have become potential fault points of UPFC system. The rapid judgment of fault state and accurate positioning of fault sub-modules are the primary conditions to ensure the safe and stable operation of UPFC system, which can effectively reduce the mobile operation and maintenance time of power engineering operation and maintenance personnel, and guarantee the safety of operation and maintenance. In this paper we proposes a fault diagnosis technology of intelligent substation UPFC system based on abnormal sub-module port voltage (SPVE). Experimental results show that the SPVE method has advantages in identification accuracy and rapidity.

\section{References}

1. Mohtashami S, Pudjianto D, Strbac G. Strategic Distribution Network Planning With Smart Grid Technologies[J]. IEEE Transactions on Smart Grid, 2017, 8(6):2656-2664.

2. Nabavi-Niaki A, Iravani M R. Steady-state and Dynamic Models of Unified Power Flow Controller (UPFC) for Power System Studies[J]. IEEE Transactions on Power Systems, 1996, 11(4):19371943.

3. Moravej Z, Pazoki M, Khederzadeh M. Impact of UPFC on Power Swing Characteristic and Distance 
Relay Behavior[J]. IEEE Transactions on Power Delivery, 2014, 29(1):261-268.

4. [9] Keri A J F, Mehraban A S, Lombard X, et al. Unified power flow controller (UPFC): modeling and analysis[J]. IEEE Transactions on Power Delivery, 1999, 14(2):648-654.

5. Gholipour E, Saadate S. Improving of transient stability of power systems using UPFC[J]. IEEE Transactions on Power Delivery, 2005, 20(2):16771682. 